ARGONE MATIOUAL LABORATORY

9700 South Cass Avenue Argonne, Illinol: 60439

STUDY OF THE DEVELORMEAT OF NEUTROA AND PLASMA RADIATIOA TEST FACILITIES

FOR THE

CTR MATERIALS PROGRAM

by

P. J. Persiani

Applied Physics Division

NOTICE

This report wa prepared is an sccount of work sponiored by the Unted States Government. Nefther the United States not the United States Atoinic Eavery. Commlation, nor eny of thetr eaptoyets, not any of their contractors, subcontractors, or thait employiat, makes any warranty, express or implied, or mumes any legel lisbility or responsibitity for the securacy, cons. pletenes of unefulnes of any inforcistion apperetus product of procen dieloced or represents the its we would not infringe privitely owned rithts.

Ju1y 1974 
Päge

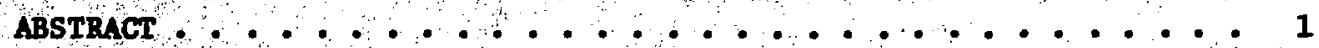

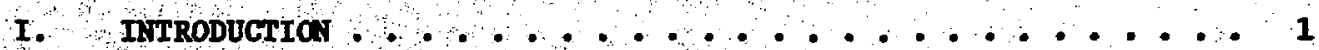

II. TEUTRON AND PLASMA SOURGE REQUIREMRITTS ......... 2

A. SURTAGE RADTATION EFTECTS. . ............ 3

B. BULK RADIATION EFTECTS . . . . . . . . . 3

III. RADIATIOI TEST FACILITIES. . . ........... 6

IV. TIMELTNESS of SOURC DEVELOPMEMT , $\ldots \ldots \ldots$

v. GEMERAL REMARKS. $\ldots \ldots \ldots \ldots \ldots$

REFERENCES $\ldots \ldots \ldots \ldots$ 


\section{LIST OF TABLES}

No.

Ittle

Page

I. Surface Radiation Studies ................... 4

II. Materials Radiation Studies ................. 5

III. Classification of (D,T) Neutron and Plasma Source Facilities. . 7

IV. Type of $(D, T)$ Neutron and Plasma Facllity - Group I . . . . . 8

v. Type of $(D, T)$ Neutron and P1agma Facility - Group II. . . . . 9

VI. Type of $(D, T)$ Neutron and Plasma Facillty - Group II-S. . . . 9

VII. Type of $(D, T)$ Neutron and P1asma Facility - Group III . . . . . 10

VII. Type of (D,T) Neutron and Plasma Facility - Group III-S . . . 11

IX. CTR Neutron and Plasma Source Concepts and Related Devices Development ..................... 14 


\title{
STUDY OF THE DEVELOPMENT OF \\ NEUTRON AND PLASMA RADIATION TEST FACILITIES \\ FOR THE CTR MATERIALS PROGRAM*
}

by

P. J. Persiani

\begin{abstract}
The Controlled Thermonuclear Research (CTR) program needs for high energy and high intensity neutron and plasma sources is investigated. The assessments include outlining source requirements, factItties avallable, and concepts for source development. The study of neutron and plasma radiation testing requirements for the CTR Materials Program Indicates the need for developing several different types of testing faclifties. An extensive R\&D program is found necessary in order to scope the feasibility of translating varlous concepts into optimum radiation testing facllities. The development must be phased Into a time scale consistent with the scheduled objectives of the overall CTR Technology Progran.
\end{abstract}

\section{INTRODUCTION}

The design of fusion devices and power reactors will be based on developing and generating neutron and plasms surface and materlals radiation design parameters. For systems based on any one of the more commonly considered fuel cycles, $D(T, n)^{4} \mathrm{He}, D(D, n){ }^{3} \mathrm{He} / \mathrm{D}(\mathrm{D}, \mathrm{p}) \mathrm{T}$ (branching ratio, 50\%), and $D\left({ }^{3} \mathrm{He}, \mathrm{p}\right)^{4} \mathrm{He}$, the plasma energy $1 \mathrm{~s}$ in the form of energetic neutrons, particles, and photons. The first two fuel cycles yield neutrons having kInetic energies of 14.1 and $2.45 \mathrm{MeV}$ respectively. In the $\mathrm{D}\left({ }^{3} \mathrm{He}, \mathrm{P}\right)^{4} \mathrm{He}$ cycle, the $(D, D)$ reaction rate 18 of the magnitude comparable to the rate of the $\left(D,{ }^{3} \mathrm{He}\right)$ reaction at plasma temperatures of 100 to $400 \mathrm{keV}$. Depending on the Initial fuel composition, the secondary and subsequent ternary reactions in the $\left(D, 3_{H e}\right)$ cycle also result in an intense source of 2.45 and $14.1 \mathrm{MeV}$ neutrons via the $(D, D)$ and $(D, T)$ chains respectively. An appreclable fraction of the fuston energy is therefore always in the form of energetic neutrons for any of the fuel systens used.

*Work performed under the cuspicse of the U. S. Atomic Energy Commiseion. 
Another component of the plasma energy is represented in the kinetic energy of the fusion reactant particles and of the unreacted primary ions from the plasma preparation phase of the fueling cycle. The charged and neutral particles are emitted from the plasma and impact on the adjacent surfaces or surface components with varying degrees of intensities via diffusion, charge exchange, recombination, and attachment processes, and from magnetic field singularities that may exist as in the case of the openended mirror systems.

The photon radiation field is comprised of plasma bremsstrahlung, synchrotron, and $X$-ray radiations incident on the containment surface and surface components; and gamma radiation generated via secondary reaction processes throughout the blanket, shield, magnet systems and other components of a power facility.

The technology of converting plasma energy into electrical energy will, therefore, be based on understanding the fundamentals of the radiation field interactions with matter in the areas such as: (1). the neutron, particle, and photon interaction effects on the vacuum wall and surface components which will influence the physics of plasma confinement, heating, and wall erosion; (2) the neutron radiation damage of materials in the form of dimensional instability (swelling), and changes in the mechanical and physical properties; and (3) the transport and transformation of the neutron and photon energy.

The exiating neutron and plasma sources fall several orders of magnitude short of simulating these radiation effects as they would occur in the planned fusion devices and power systems.

The purpose of this study $(1,2)$ is to anticipate the needs and the necessary development for neutron and plasma source testing facilities in the CTR Technology program. The study includes surveying, technically assessing and evaluating the surface and materials programmatic needs and the capabilities of exdsting and proposed source facllities to satisfy these needs.

\section{NEUTRON AND PLASMA SOURCE REQUIREMENTS}

The energy and inte:nsity requirements of the source are varied and involve generally seven a:eas of experimental study. These are 1isted below In an approxlmate order of increasing source intensity.

CTR NEUTRON AND PLASMA SOURCE REQUIREMENTS

1. Cross Section and Related Nuclear Data

2. Superconducting Sys tem Materials Studies

3. Radiolysis in haterials

4. Engineering Design-related Neutronics

Experiments (Integral Experiments)

5. Surface Radiation Studies

6. Materials Radiation Studies

7. Engineering Radiation Experiments

The first four areas do not appear to impose severe demands on source energy spectra and/or intensity and therefore can probably be satisfied by avallable or modified aystems within current technology. 
The experimental program needs for the latter three areas require from modest to extensive research and development efforts in scaling concepts into more intense testing facilities.

In assessing the technical justification and requirements for Radiation testing facilities, it is necessary to delineate between two major areas; the (A) Surface Radiation Effects, and (B) Bulk Radiation Effects.

(A) Surface Radiation Effects

The surface effects involves the study of the primary and secondary plesma radiation (MeV neutrons, energetic particles and Ions, X-rays, bremsstrahlung, soft $X$-rays and synchroton radiation) interaction effects on the immediately adjacent vacuum-wall surface components. The resulting blistering, sputtering, and particulate emission lead to wall erosion and plasma contamination, which in turn influences the physics of plasma confinement and heating. A most important aspect of the overall surface area problem is that the incident radiation field is a spectrum of reaction mechanisms. The surface problem relates directly to the plasma physics and confinement experiments such as the Fusion Test Reactors being planned in the major CTR laboratories. The importance of these experiments places a priority of efforts in this phase of CTR program.

The more long range consequences of plasma-wall interactions will include the effects on the physical and structural integrity of surface components including electrical properties of insulators relating to the conditions in fusion power reactors.

A summary of the measurements description for surface effects experiments and facility requirements are presented in Table I.

\section{(B) Bulk Radiation Effects}

The bulk radiation effects involves the scudy of fusion spectra ( $\mathrm{eV}$ to $14 \mathrm{MeV}$ ) neutrons with the structural component materials of higher power fusion reactors. The volume integrated (bulk) neutron fluence effects on the physical and mechanical properties, creep strength, ductility and dimensional instability (swelling) relate to the design problems of prototype and/or demonstration fusion power reactors. The high performance conditions associated with the power reactors necessitates initiating basic materials studies, neutron and ion-bombardment correlation experiments, void formation, defect formation, etc.

The bulk radiation measurement descriptions with neutron source strength requirements are outlined in Table II. The test conditions for the bulk studies require neutron fluxes and fluences of one or two orders of magnitude greater than the needs specified in the surface science program. The minimum fluence needed to obtain observable measurements for surface effects ( $10^{27}$ to $10^{18} \mathrm{n} / \mathrm{cm}^{2}$ ) is lower than the minimum fluence requirements for messuring observable bulk effects $\left(>10^{20} \mathrm{n} / \mathrm{cm}^{2}\right)$.

Neutron source facilities in the energy range and intensities of long term interest to the CTR program, are presently not avallable. 
Table I

\section{Surface Radietion Studies}

\section{A. Measurement Description}

(1) Vacuum wal1, surface components, and insulators erosion

(a) Sputtering

(b) Radiation blistering by photons, particles, neutron and reaction products: $(n, \alpha),(n, p)$, etc.

(c) Particle desorption by direct plasma radiation, neutron and reaction product interactions.

(d) Photo-decomposition of surface compounds by plasma and by neutron-induced energetic photons and reaction products.

(e) Radiation damage in surface layers.

(2) Plasma contamination

(a) Multiple backscat tering.

(b) Secondary particle emission (see 1.(a)-1.(d) above).

(c) Secondary electron emission (electron sheath formation).

B. Source Description

Plasma Density:

Neutron Energy Range:

Intensity (at sample surface):

Fluence (yield 0.1 monolayer):

High Fluence Effects:

Geometry :

$$
\begin{aligned}
& 10^{16} \mathrm{p} / \mathrm{cm}^{3} \\
& \mathrm{keV}-14 \mathrm{MeV} \\
& >10^{12} \mathrm{n} / \mathrm{cm}^{2}-\mathrm{sec} \\
& 3 \times 10^{17} \mathrm{n} / \mathrm{cm}^{2} \\
& 10^{20}-10^{21} \mathrm{n} / \mathrm{cm}^{2} \\
& \text { point source, beam flux }
\end{aligned}
$$


Table II

\section{Material Radiation Studies}

A. Measurement Description

(1) Neutron fluence effects on physical and mechanical properties: creep strength, and 108 s of ductility in vacuum wall and st uctural material at temperatures in range of $500-1000^{\circ} \mathrm{C}$.

(2) Synergetic effect of high gas generation and point defect production rates in a high flux of high energy neutrons on void formation at temperatures in the range of $500-1000^{\circ} \mathrm{C}$.

(3) Establish correlation between heavy-ion bombardment effects and neutron radiation effects at several energies (descrete or integral) in the range of $2-14 \mathrm{MeV}$.

(4) Transmutation effects on physical and mechanical properties of structural materials.

B. Source Description

Energy Range:

Intensity (long term):

H1gh Fluence:

Intensity (correlation experiments) : Minimum Fluence:

(irradiation period 4 mo., 2 wk.) Geometry:
keV-14 MeV

$>10^{15} \mathrm{n} / \mathrm{cm}^{2}-\mathrm{sec}$

$10^{23}-10^{24} \mathrm{n} / \mathrm{cm}^{2}$

$10^{13}-10^{14} \mathrm{n} / \mathrm{cm}^{2}$

$10^{20} \mathrm{n} / \mathrm{cm}^{2}$

point source, beam flux

in-core irradiation 


\section{RADIATION TEST FACILITIES}

Many devices such as solid and liquid targets, tritium drive-in targets, gas-targets, dense plasma focus devices, laser-plasma, e-beam plaswa devices as well as magnetic field confinement devices have been considered as potential candidates for developing into intense neutron and plasma generators. The variety of these concepts and the variety of the CTR programnatic needs necessitated introducing some order of classification.

A preliminary classification involves five groups as outined in Table III. The three main groupings are based, in part, on the type of systems and the level of source intensity actually achieved or anticipated. The supplementary Group II-S and III-S are defined to indicate that the concept may be utilized as an auxiliary heating technique for other systems as well as a source device itself. Group I and part of Group II are neutron sources whereas the remalning concepts are neutron and/or plasma sources.

The general design features and problems and the anticipated contribution to the surface, materials and engineerting program for each of the categories have been reviewed and involve: the level of intensity; source geometry; a statement on the effort required for the successful development of the concept; the relationship between the activities involved in developing the source and the activities involved in the CTR program; and an estimate of the scale of the necessary research and development for each of the categories. A summary of these considerations are included in Tables IV-VIII.

\section{TIMELINESS OF SOURCE DEVELOPMENT}

There has recently been an increasing emphasis among plasma physicists to understand and control impurities resulting from plasma radiation and wal1-surface interaction. (3-9) The impurities have the effect of increasing the radiation losses of the plasma and of introducing instabilities which enhances the diffusion of the impurities into the plasma and compromises the confinement. These impurity effects are found to be strongiy species dependent. It is therefore important to correlate the mechanisms, energy spectra, and types of surface impurities with the type of radiation fields in both energy and intensity. This dependence on the quality of the impurity as a function of the incident complex radiation field of a plasma, introduces a need for developing test facilities based on a plasma source concept. The plasma problem will be of central interest in the near-term (5-8 years) plasma physics and confinement program. The completion of the goals and objectives in the CTR Program will depend on the understanding of the plasma-wall interaction. The program of experiments outilned by the surface investigators is central to the investigation of these problems. In addition to the experiments 
Table III

Claselfication of (D,T) Neutron and P1esen Source Fecilities

Group I - Accelerator and Target Syetem

1. Rotating target system

a. Solld $\mathrm{TiT}_{2}$ target

b. Sputtering-electrode $\mathrm{T}_{\mathbf{1}} \mathrm{T}_{2}$ thick target

2. Drive-1n target system

a. Mixed (D,T) Ion beam on solid $C r$ target

3. Self-replenishing target system

a. Tritium gas flow in solid $\mathrm{TIT}_{2}$ target

4. Liquid-jet LiT/LI mixture target

Group II

1. Dense plasma focus device

2. Supersonic gas-jet target

3. Liquid-jet target (LiT)

Group II-S

1. Laser-plasma Interaction device

a. Auxdliary heating for Group III type concepts

b. Laser-plasma solenold as neutron and plasma source

2. Relativistic-electron-beam plasma Interaction device

a. Auxiliary heating for Group III type concepts

b. Relativistic-electron-beam plasma as neutron and plasma source

3. Drift Orb1t Cyclotron Heating (DOCH)

a. Auxillary heating for Group III type concepts

Group III

1. Theta-Pinch Machine

2. Mirror-Machine

3. Others

Group III-S

1. Laser-pellet interaction

2. Relativistic-electron-beam-pellet interaction 
Table IV

Type of (D,T) Neutron and Plagma Fact11ty

Group I

Deslgn-related Considerations

1. Low Intensity $>10^{13} \mathrm{n} / \mathrm{sec}$

2. Polnt geometry source

3. LImited experimental access

4. Low calculated rlsk in performance expectation

5. Modest scallng for accelerator and target development
6. State of art technology

7. Technology for source development not related to the main CTR development program in the SFX

8. Moderate Investment in R\&D (mil11ons)

\section{Ant1cipated Contribution to Materials Program}

1. Inftiate scoplng experiments on effects of fusion-energy neutrons on surface and materials.

2. Observation of threshold or onset of damage phenomena.

3. Assess signiflcance of damage phenomena.

4. Guidance in planning a surface and materials development program directly relevant to fusion reactor systems.

5. Contribute to establishing and planning specific output feature of the CIR Radiation Testing Facility. 
Table V

Group II

Design-related Considerations

1. Medium to high intensity $>10^{14} \mathrm{n} / \mathrm{sec}$

2. Point source geometry

3. Limited experimental access

4. Higher calculated risk in performance expectations than 9. Major investment In R \& D in Group I
6. Hajor R \& D effort for developent

7. Extending current technology

8. Technology for development peripherally related to main CTR program for SEX

(tens of mil11ons)

5. Moderate to substantial scaling

Anticipated Contribution to Materials Program

1. Surface Radiation effects at higher fiuence.

2. Signiflcant radiation fluence allowing study of materials damage over extended time periods.

3. Improved assessment of damage phenomena (dimensional instability) for preliminary design studies.

4. Neutron fluence effects on mechanical and physical properties for structural materials.

Table VI

Group II-S

Design-related Considerations

1. Medium intensity as a neutron 5. Substantial scaling required source

2. Point, 1ine, or extended source geometry

6. Major R \& D effort for development

7. Extending current technology

3. Alternate function to use as

8. Technology for development marginauxillary heating in Group III and III-S ally related to the main CTR program

4. Higher calculated risk in performance expectation than Group I type

\section{Anticipated Contribution to Materials Program}

1. Indirectly as an auxillary heating subsystem of a neutron and plasma source.

2. As a source same as Group II systems. 
Table VII

Group III

Design-related Considerations

1. Medium to high flux $>10^{14} \mathrm{n} / \mathrm{cm}^{2}-\mathrm{sec}$

2. Extended geometry

3. Extended accessibility to accommodate a range of experimental program

4. Lower calculated risk than "break-even" thermonuclear machine

5. Substantial scaling required

6. Najor R\&D effort for development can be obtained from the main

CTR SFX program
7. Extending current technology

8. Technology for development of a thermonuclear machine as a neutron source is related directly with the main CTR program

9. Major extensive investuent in $R \& D$ (Facility level effort) Added note: The current SFX RED progran forms the basis for development of the fusion radiation test facility

\section{Anticipated Contribution to Materials Program}

1. Surface and Materials radiation effects and Engineering Testing Progran In environment characteristic of a fusion power reactor.

2. Engineering testing program in this environment includes:
a. materials danage
b. blanket designs
c. superconducting ays tem design
d. Instrumentation for reactor control

e. 1sotopic concentrations

f. 1iquid-wetal coolant chenietry

g. heat traneport wechaniew

h. vechanical detign:

1. etc. 
Table VIII

Group III-S

Design-related Constderations

1. Medium to high flux

$$
>10^{14} \mathrm{n} / \mathrm{cm}^{2}-8 e c
$$

2. Extended geometry

3. Extended accessibility to acconmodate a range of $e \ddot{x}-$ perimental programs

4. Lower calculated risk than "break-even" thermonuclear machine

5. Substantial scallng required
6. Major RED effort for development

7. Long-range technology and development

8. Technology for development related directly with laverinduced fusion CIR progran

9. Major extensive Invertment in RED

\section{Anticipated Contribution to Materials Program}

1. Surface and Materials and Engtneering Testing Program in environnent characteristic of a laser-induced fusion power reactor. 
utilizing currently avallable or planned test facilities, the surface program Identifies the need for experiments with total radiation fields more characteristic of a thermonuclear plasma. The purpose being to study the synergetic effects of $\mathrm{MeV}$ neutrons, particles, bremsstrahlung, soft $\mathrm{X}$-rays and synchroton radiation.

The plasma source has the potential of simulating a thermonuclear plasma radiation environment and as such would be a major advance in the level of surface and bulk radiation research which can be performed. The simulation relates to the condition that in thermonuclear plasma reactions the relative intensity ratios of the neutron and alpha particles, and the bremsetrahlung radiation are constant and Independent of the type of confinement device. The relative ratio with respect to synchrotron radiation 18 somewhat device dependent and will rely on estimating the opacity of the plasma to this radiation. A further correlation between the plasma test source and thermonuclear confinement machines may also be established from the presence of a plasa and magnetic field Interface region in both systems.

The timeliness of neutron and plasima source requirements introduces a new emphasis in considering the general area of Surface Radiation Effects. The near-term needs emphasize the plasma radiation and wall surface Interaction as it relates to the plasina confinement progran. The long-ter needs relate to the plasma-wall interaction effects on the physical and structural integrity of the surface components under Fusion Power Reactor conditions. This is aumarized as follows:

Surface Radiation Effects

A. Near-Term Needs

A.1 Plasma Radiation and Wa11 Surface Interaction

A.2 Plasma Physics Confinement Progran

A.3 Understanding Separate and Cosbined Radiation Component rachaniam

A.4 Controlling Plasia Containation

B. Long-Term Needs

B.1 Plasma-Wall Interaction Effects on Physical and Structural Integrity on Surface Components inder Fusion Power Reactor Condition

The bulk radiation problen will becone elgnificant for the longer-ter high-performance fusion power reactor system. The fluxe and fluence of MeV neutron required by this progran are wore severe than the inteneity requirements of the surface studies at this time. The neutron source concepts that have been suggested would partially satisfy the bulk anterials progran. The limitation se to type of experinente and/or significance of the results of these experimente to the CTR progran are a consequence of the fluance capabilities of these nachines, and just as important the linitation resultIng from the amall-size saple acces capability and the position of peak flux. 
The time-phase in neutron source development assoctated with the Bulk Radiation Effects may be outlined as follows:

Bulk Radiation Effects

A. Near-Term Needs

A.1 Low Fluence for Basic Materlals Studiea and Analytical Kodeling Correlations w1th Ion-Bowbardment Techniques

B. Long-Term Needs

B.1 High Fluence Radiation Danage for Structural Integrity in Durion Power Reactor Conditions

Engineering-Type Experiments

For Englneering-Type Radiation Experinents, the tine-enphasis is longterm for large-scale experiments of the many subsystem in a Fusion Power Reactor. Eventually the study of surface and bulk radiation effects as well as large-scale engineering tests relating to the high-performance condition of fusion power aystems, w11 require testing facilities on the level of a Fusion Engineering Research Facility, FERF. Prelininarg feasibility design studies on FERF type systems are presently being pursued by several CIR laboratories.

The time-emphasis of source development and the wain CIR Progran can be assessed by the schedules outlined in Table IX. Neutron sources ay be developed in time to influence el ther the experimental progra or the design of several near-term confinement experiments. The internadiate plana source In addition to its utility as a neutron source may contribute importantly to the FTR-1 and 2, and EPR design programs, if developed in tine.

\section{v. GENERAL RRAARKS}

The selection of neutron and plasen source concepts for developins into radiation test facilities must be based on the following conideration:

(a) The level of source intensity actually available to the investigetor;

(b) The capatility and flextbility of the source concept to accomodate a range of experimentel needs;

(c) The number of experiments which can be perforned almultineouls in order to provide a broad base of aupport for the total facility:

(d) The experfiental date output from the facility is of the quality output needed to anke significant advance in understanding radiation interaction with enterials; and

(e) The econonics of the research and development neceasary to establish the feasibility of a concept, the capital coste to build the facilty, and the operating coste per experiment.

An important phase in the logiatica of developing Rediation Teat Facilities is the formulation of an overall CIR-Technology experimental progra. 
CTR Mutron and Plasm Source Concepts and Related Devices Development

$1974 \quad 1975 \quad 1976 \quad \underline{1977} \quad 1978 \quad \underline{1979}$

Heutron Source

Rotating Target R.T. (Li:)

RT Ia

RT II

Super Sonic Jet-Target (LASL)

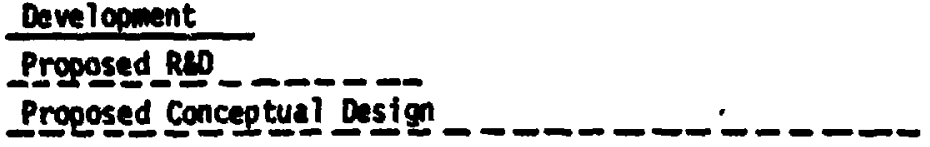

Plasm Source

Plasan Focus DPF (EURATOH)

Developinent

Laser-Beam-Plasma Interaction

E-Basim-Plasna Interaction

Other Plasma Concepts

Plasm Device (Fusion Engineering Radiation Facility)

Mirror

O-Pinch

Conceptual Design

CTR Plasma Confinewent Progran

Princeton PLT

Princeton PDX

LASL SYLLFC

LL Small Hirror - 2K-IIB (Pulsed)

LLL Mirror Baseball II, HX $I_{*}$ 2X-II Advanced

GA Doublet III

Conceptual Design

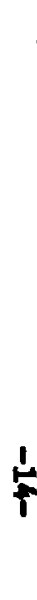

Fusion Test Reactor (FTR) FTR-1 (Tokamak Q-T Burner)

FTR-2 (Nirror or כ-Pinch D-T \&urner)

EPR (Experimental Power Reactor)

$\frac{\text { Developinent }}{\text { Experimental Program }}$


The Identification and delineation of the specific need of this broad eurface and bulk enterials radiation progran and engineering teet progran 111 be the mijor factor In Influencing the selection and design festures of the optinu test facilities. The coordination of designer's efforts with users' needs would require that a Designers-Deers Group include the following responsibilitien:

(a) Identifying, evaluating and coordinating the interface problea area of various disciplines Involved In the design and the uers' neede into a studied effort toward the soel of developins a radiation fecillty,

(b) Scoping the experimental progran which will Influence the dealgn features,

(c) Inttlating and developing new areas of effort necesary to resolve problew within each of the disciplines as well. as acroas the disciplines,

(d) Evaluating the technical ignificance of developents and progress in other CTR programs and translating these into the design of the facility,

(e) Mantaining an overview of the total progra in order to put into better perspective the balance between the angnitude of effort and the quality of the output for program and eub-profecte,

(f) Utiliz:,ig experience or knowledge in the field of research renctor desigr., accelerator deslgn, anget oystem, energy storage and transfer systems, etc. 


\section{References}

1. P. J. Peraiani, "Weutron Source Consideration for the CrR Materials Progran", Proc. of Workshop on High Intensity Neutron Cenerators, Las Vegas, Nevada, June 1972. p. 111.

2. P. J. Persiant, "A Repiort on the Development of Intence Neutron Source Bacilities for the CTR Technolosy Progran", ANL/CIR-73-01, August 1973. To be published in Nuclear Fuilon.

3. B. Copp1, H. P. Furth, M. M. Rosenbluth, and R. Z. Sagdeev, "Drift Instab1fty Due to Inpurity Ions", Phys. Rev. Lett. Vol. 17, (1966), p. 377.

4. E. P, Gorbunov, V. S. Zaverjaev, and H. P. Petrov, "Behavior of Ions in the Tokanak-4 Plasma", Proc. S1xth European Conf. on Cont. Pusion and Plesen Phyelce, Howcow, USSR, Vol I, July-Auguet 1973, p. 1.

5. F. Devirco, "Measuresent of the Inpurity Level in the ATc", Bul1. An. Phys. Soc., Vol. 18, (1973), p. 1253.

6. S. Von Goeler, W. Stodiek, and H. Sauthoff, "Impurities in the ST Tokanek, IBID, P. 1254.

7. E. Heservey, K. Bretz, D. Dinock, and E. Hinnov, "The Effects of Light and Heavy Inpurities on a Tokmak Discharge", IBID p. 1254.

8. B. Coppt, "Plasan Nodes Due to Impurity and Magnetically Trapped Ion", Phys. Rev. Lett. Vo1. 31 (1973), p. 1443.

9. T. Kamanh and D. L. Galbratth, "Inpurity and Injection Eneroy Effecta on Toroldal Reactor Dynanice", Proc. Firet Topical Heeting on the Technology of Controlled Kuclear Fusion", ANs, San Diego, California, Apri1 16-18, 1974., p. 120. 